\title{
Micronización de las áreas de interés del proceso global de enseñanza-aprendizaje en prueba de fin de curso: licenciatura en nutrición de la Universidad Autónoma de Tamaulipas
}

\author{
Nali Borrego*, Ma Del Rosario Contreras, Marcia L. Ruiz y Rogelio Castillo \\ Universidad Autónoma de Tamaulipas. Matamoros SN, Zona Centro Ciudad Victoria, Tamaulipas México. \\ (nborrego@docentes.uat.edu.mx; mcontrer@docentes.uat.edu.mx; mruizc@docentes.uat.edu.mx; \\ rocastill@docentes.uat.edu.mx)
}

${ }^{*}$ Autor a quien debe ser dirigida la correspondencia

Recibido Jun. 16, 2021; Aceptado Ago. 10, 2021; Versión final Sep. 28, 2021, Publicado Feb. 2022

\begin{abstract}
Resumen
El objetivo de este estudio es llevar a cabo un proceso de simplificación para micronizar áreas de interés del proceso global de enseñanza-aprendizaje en la evaluación de fin de curso de 27 asignaturas de la licenciatura en nutrición. Participan 270 alumnos de la Universidad Autónoma de Tamaulipas (México). Se hace una intervención en tres fases que posteriormente se convierte en un proceso: 1) antes (la preparación), 2) durante (la aplicación), y 3) después (la aplicación de test y retest). Posteriormente, se integra la fundamentación didáctica micro-orientada de las áreas de interés. El análisis revela una aceptable modelación de calificaciones y áreas de interés que aporta elementos prácticos para la formulación del concepto de microevaluación. Se concluye que esto contribuye a la innovación de la prueba de fin de curso con alcance en la preparación de los alumnos para el examen EGEL (Examen General de Egreso de Licenciatura) de México.
\end{abstract}

\section{Micronization of global teaching-learning-process interest areas in final exams of the bachelor's nutrition degree at the Autonomous University of Tamaulipas}

\begin{abstract}
The main objective of this research study is to conduct a simplification process to micronize interest areas of the global teaching-learning process. This is performed to assess the final exams of 27 courses of the bachelor's nutrition degree at the Autonomous University of Tamaulipas (Mexico). A total of 270 students participate in the study. A three-phase intervention is applied and converted into a multi-step process: 1) before (preparation), 2) during (application), and 3) after (test application and retest). A micro-oriented didactic rationale of the areas of interest is integrated based on Stanford's criteria and instructional events. The results reveal an acceptable modelling for grades and also show areas of interest that provide practical elements for the formulation of a micro-assessment concept. It is concluded that these findings contribute to innovating final course exams while preparing students for the EGEL (Examen General de Egreso de Licenciatura) exam in Mexico.
\end{abstract}




\section{INTRODUCCIÓN.}

En los años 90, el Banco Mundial expresó su preocupación por el deterioro de la calidad de la educación superior, especialmente en América Latina, y lo atribuyó al aumento de la matrícula. Entre las soluciones que planteó están las relacionadas con la evaluación. En la actualidad las políticas educativas latinoamericanas son fuertemente influenciadas por los mandatos de organismos de control globalizados, el propio Banco Mundial, la Organización para la Cooperación y el Desarrollo Económicos (OCDE), la Organización de las Naciones Unidas para la Educación, la Ciencia y la Cultura (UNESCO). En el ámbito latinoamericano por el Banco Interamericano de Desarrollo (BID), la Comisión Económica para América Latina (CEPAL), la Organización de los Estados Americanos (OEA), Organización de Estados Iberoamericanos (OEI) y un número incalculable de Oenegés (ONG) que actúan en relación con la financiación asignando un papel central a la evaluación (Galazzi et al., 2019). Los procesos de evaluación involucran de manera puntual a profesores, instituciones y alumnos de la educación superior (González-Barea et al., 2021).

Esta es una de las razones por las que la evaluación tradicional de los alumnos se centra en los resultados y no en los procesos que conducen al aprendizaje o en la construcción personal del conocimiento, lo que tiene un impacto negativo en el aprendizaje. Sepúlveda et al. (2020) realizaron una revisión de importantes estudios recientes que les permitió articular una alternativa en la que prevalecen varias funciones, como: el objeto evaluado, el rendimiento, la verificación de los objetivos, la valoración de los resultados, la clarificación de los objetivos y su reformulación. Toman como punto nodal la creación del concepto de evaluación para el aprendizaje impulsado por la idea de que logra una especie de autorregulación del aprendizaje de los alumnos, y que además promueve el desarrollo cognitivo, la creatividad y la comprensión para poder resolver problemas. Lo relacionan con los objetivos, refiriéndose a la evaluación para el aprendizaje que consiste en proporcionar información a la comunidad educativa sobre las formas de mejorar la calidad de los procesos de enseñanza aprendizaje, y lo añaden a la finalidad principal de la evaluación, que es el aprendizaje, sus dificultades y el proceso de enseñanza basados en la metaevaluación. Finalmente concretan el concepto de evaluación de la evaluación a través del cual la comunidad educativa comprende su sentido, aprende a optimizar y a ajustar mejor las formas en que evalúa y se autoevalúa, concluyendo que la evaluación frecuente de las evaluaciones debe ser una constante.

Entre algunas estrategias, la microenseñanza se identifica como impulsora de la evaluación en el aula y que ha dado lugar a una familia de micros. Es una metodología utilizada en los laboratorios de docencia desarrollada en la Universidad de Stanford en 1960 (Allen, 1966). También origen del microlearning o microaprendizaje empleado en la formación del profesorado para complementar la teoría, proporcionando una experiencia a pequeña escala en la que aprenden nuevas técnicas con tres o cuatro alumnos y lecciones cortas de cinco y no más de quince minutos, sin dañar el aprendizaje de los participantes. Raíz de la microdocencia Dulsat-Ortiz (2019), así como del modelo microcurricular activo de Posso-Pacheco et al. (2020). Esto se interpreta como transposición didáctica, que consiste en el paso del conocimiento conocido al conocimiento enseñado que se busca en las prácticas de laboratorio de microenseñanza (Chevallard, 2007).

La metodología es ampliamente aceptada en la formación de profesores, y profesionales de la logopedia, la psicología, la comunicación, las ciencias biológicas, la formación médica, en la que se simulan situaciones del futuro profesional para acercar a los participantes a lo que pueden encontrar en escenarios reales (Dulsat-Ortiz, 2019). Como las escenas de microlearning útiles a la socialización mediática y las identidades lúdicas que lo convierten en multisensorial Hug (2007) y multimodal Dolasinski y Reynolds (2020), adecuado para satisfacer la creciente demanda de desarrollo profesional continuo resultante de los cambios en el lugar de trabajo, donde los procesos tradicionales no son suficientes y las micro metodologías en entornos en línea, como los medios sociales, son eficaces. (Kelleci et al., 2018). Sin embargo, la evaluación ha sido solo un componente de estos procesos, lo que también sucede en entornos donde se usa el papel, y se prefieren pruebas escritas al final del ciclo que no contribuyen al desarrollo de habilidades cognitivas superiores, solo cumplen con la norma Sepúlveda et al. (2020), son condiciones que instan a realizar un ejercicio de transposición didáctica en el proceso de evaluación, razón para recurrir a experiencias innovadoras entre las que destaca la evaluación gamificada.

En ese sentido, cabe señalar que, al observar la corriente principal de la gamificación consolidada en el año 2010, la evaluación gamificada de los estudiantes universitarios se ha posicionado de forma sobresaliente (Tsay et al., 2018). Existen varios estudios notables en los que se manifiesta un alcance más allá de las puntuaciones, las insignias, las tablas de clasificación y muestran evolución hacia algo con más poder para facilitar el aprendizaje (Huang et al., 2020). Por ejemplo, los estudiantes universitarios siguen trabajando incluso cuando han alcanzado el máximo número de puntos de calificación, considerado este comportamiento como una evidencia de compromiso cognitivo (Ibáñez et al., 2014). Los estudiantes que no participaron en el trabajo del curso gamificado sienten que pierden la oportunidad de lanzar su empresa e interactuar con las organizaciones que les favorecen, y las notas finales son más altas para quienes participan en las secciones del curso que utilizan gamificación (Isabelle, 2020). Mejora los hábitos de salud al actuar sobre la motivación a través de una intervención gamificada para la evaluación de un programa de educación para la salud (Pérez, 2017). 
También surge la evaluación gamificada no digitalizada para enseñar matemáticas y mejorar las calificaciones (Zabala-Vargas et al., 2020). El escape room presenta mayor potencial que una metodología tradicional en Matemáticas (Fuentes-Cabrera et al., 2020). El juego de roles con estudiantes de contabilidad que se enfrentan a una situación empresarial en el aula influye de manera positiva en las calificaciones (González-Acosta et al., 2020). Como variante de la evaluación gamificada en la educación superior, se perciben las aplicaciones móviles que apoyan la motivación dentro del aula y facilitan la conexión con el proceso (Morales et al., 2020). El uso de la aplicación de vocabulario gamificado para estudiantes universitarios que participan en la construcción de un repertorio de vocabulario (Kohnke et al., 2021). De la aplicación Kahoot se informa de aproximadamente 1500 publicaciones en las principales bases de datos y en diversos repositorios institucionales, la mayoría de las cuales apoyan el relevante posicionamiento de la evaluación gamificada dentro de la corriente principal de la gamificación, revelando que en ella se nivelan las fuerzas del trabajo escolar, sin sustituirlas, y se aportan beneficios adicionales sin constituir un fin en sí misma.

Al utilizar una mecánica de juego o una amplia variedad de mecánicas de juego, bien, sea dedicadas al entretenimiento, a generar aprendizaje significativo, a enseñanza de un área específica o simular situaciones del mundo real, en cualquier caso, se corre el riesgo de que los estudios evaluativos se reduzcan a un nivel instrumental. Teniendo en cuenta esas consideraciones, en este estudio se asume la gamificación basada en proceso de cambio, de tiempo y de contenido entre otras, y actividades de motivación, de significación, de compromiso, de competencia, de trabajo en equipo (Sailer y Sailer, 2021). En esta lógica, la visión de la evaluación para el aprendizaje, la evaluación de la evaluación o la metaevaluación se comparte con Sepúlveda et al. (2020) y se reconoce como proceso.

La gamificación aplicada a la evaluación destaca en algunas formaciones, como es el caso de la formación del profesorado (Castañeda-Vázquez et al., 2019). La formación en pedagogía infantil (Jaimes, 2020). La formación del profesorado de pedagogía (Fuentes-Cabrera et al., 2020). Con el mismo fin, la microenseñanza también tiene lugar en la formación del profesorado Dulsat-Ortiz (2019), en algunos campos ya mencionados: la logopedia, la psicología, la comunicación, las ciencias biológicas y la formación médica entre otras. Son dos enfoques metodológicos que pueden contribuir a la mejora del proceso de evaluación, a pesar del significativo desafío que esto representa, porque las áreas de interés del proceso global de enseñanza-aprendizaje están vinculadas a factores pedagógicos, institucionales, sociodemográficos y psicológicos entre otros, y tradicionalmente la evaluación se encarga de responderles por medio de calificaciones. Para cumplir con ese objetivo la Universidad Autónoma de Tamaulipas prepara los estudiantes para el Examen General de Egreso (EGEL) capacitando a los docentes en la elaboración de reactivos y lograr una mayor cantidad de aprobados que permita proporcionar a la comunidad información confiable sobre el aprendizaje alcanzado por los estudiantes, cabe señalar que la licenciatura objeto de estudio cuenta con su propia guía (Nutrición - Ceneval, 2021).

Con base en lo anterior y ante la dificultad de hablar de proceso en términos de evaluación, se propone una intervención de la evaluación en tres fases: el antes, el durante y el después para obtener información sobre el rendimiento del estudiante que comprende la presencia de dispositivos derivados de una meta a alcanzar, de las reglas para determinar cómo lograr esa meta, de un sistema de retroalimentación sobre información del nivel de progreso y participación voluntaria (Alhalafawy y Zaki, 2019). Así como obtener informes del aprovechamiento, un aspecto importante en el proceso de finalización de tareas de la gestión del tiempo dedicado o el uso efectivo de ese tiempo que tiene efectos positivos en el éxito académico y el logro de la sesión de evaluación. Y de la motivación, relacionada con el compromiso del estudiante, considerada una parte esencial del proceso de realización de las tareas donde el grado de interés, y el grado de motivación ante una prueba de evaluación pueden determinar el éxito. De la ubicuidad con relevancia en los procesos escolares motivada por el desarrollo tecnológico, derivada del concepto de "sociedad de la ubicuidad" adherida por medio de las tecnologías de la comunicación y la información (Liu et al., 2018). En el presente estudio específicamente a la ubicuidad de la prueba está limitada por la norma donde no se contempla la prueba en casa, convirtiéndose en un tema especial y en un asunto de decisión del profesor y del estudiante. Por último, la satisfacción escolar, directamente relacionada con el rendimiento académico (Gutiérrez-Monsalve et al., 2021). Reducir las áreas de interés del proceso global de enseñanza aprendizaje en la prueba de fin de curso, significaría mejorarla ya que el propósito de la micronización y la gamificación es incidir de forma positiva en los procesos.

\section{MÉTODO}

Se implementó la intervención en la prueba no digitalizada y la prueba digitalizada gamificada para recabar información y realizar un análisis categorial de la percepción de los estudiantes que cursan la Licenciatura en Nutrición en la Unidad Académica de Trabajo Social y Ciencias para el Desarrollo Humano de la Universidad Autónoma de Tamaulipas. Se trata de la prueba reglamentada de fin de curso que equivale al $50 \%$ de la calificación global de cada asignatura en la que se propone indagar si existen condiciones para la micronización de las áreas de interés del proceso global de enseñanza aprendizaje en la evaluación de fin de curso. Se plantean dos hipótesis: $\mathrm{H}_{0}$ al presentar dos formatos de prueba, y uno de ellos poco usual, no se 
encontraría diferencias significativas y $\mathrm{H}_{1}$ al presentar dos formatos de prueba, y uno de ellos poco usual, se encontraría diferencias significativas.

El estudio se sitúa en una perspectiva empírico-analítica de corte cuantitativo cuasiexperimental, con diseño de un grupo único con tratamiento de test-retest que el grupo responde la misma prueba dos veces en un intervalo de tres días durante una semana. Los métodos usados son el descriptivo, el correlacional tanto para describir las relaciones entre las distintas áreas de interés como para interpretar el cambio. Al iniciar la investigación basada en proceso micro orientado, se identificó un incremento en el número de búsquedas de términos con prefijo micro relacionado con el aprendizaje durante los últimos cuatro años según Google Trends (Figura 1). El término más buscado es microlearning, seguido de microenseñanza y microaprendizaje, lo que indica que hay un creciente interés en esta metodología.

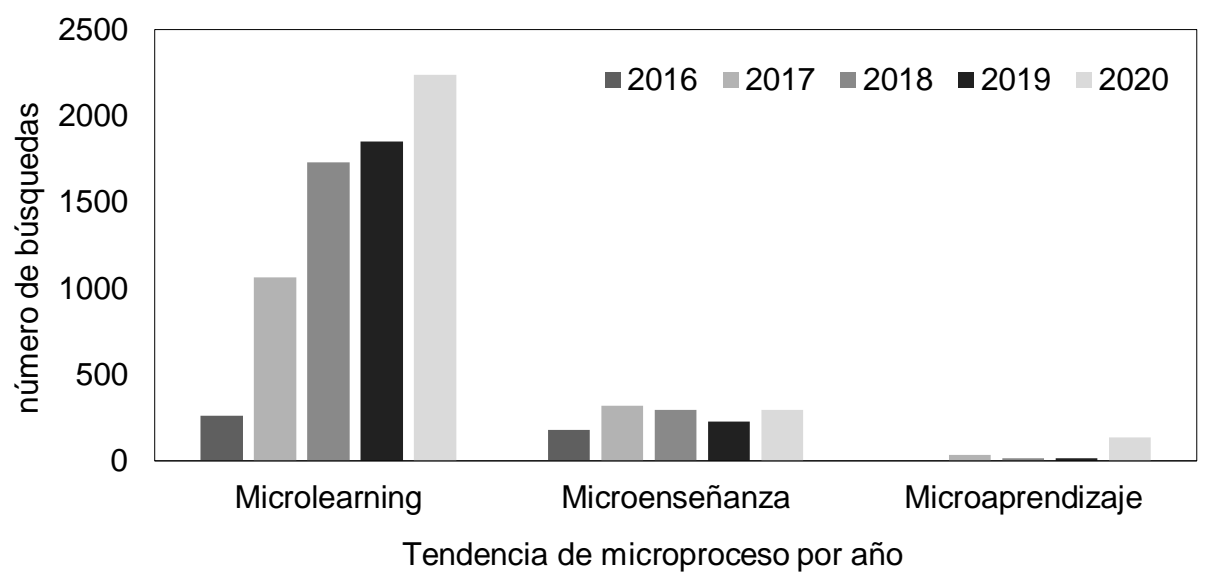

Fig. 1. Tendencia de búsqueda de microlearning, microenseñanza y microaprendizaje en los últimos cuatro años.

\section{Muestra}

La muestra es de 270 estudiantes de 27 asignaturas de la Licenciatura en Nutrición, en el reglamento de la UAT se establece que se deben presentar dos pruebas, una parcial y otra al final del curso, la segunda es objeto del estudio. Para el muestreo se utilizaron dos técnicas probabilísticas, primero se aplicó la técnica de muestreo aleatorio estratificado en la Tabla 1, el total de alumnos por asignatura que debían presentar el examen final, y la cantidad de estudiantes con probabilidad de participan en el estudio por grupo y asignatura.

Tabla 1. Universo y muestra estratificada de estudiantes que asistieron a realizar el examen final.

\begin{tabular}{|l|c|c|c|c|c|}
\hline \multicolumn{1}{|c|}{ Número de Asignatura } & Período & Población & \%Universo & $\begin{array}{c}\text { \% } \\
\text { Muestra }\end{array}$ & $\begin{array}{c}\text { Cantidad } \\
\text { Sujetos }\end{array}$ \\
\hline 1.Seminario de Investigación en Nutrición II & $8^{\circ} 1$ & 10 & 2.73 & 3.37 & 7 \\
\hline 2.Matemáticas Básicas & $1^{\circ} 1$ & 11 & 3.01 & 8.12 & 8 \\
\hline 3. Programas de entrenamiento y ejercicio físico & $6^{\circ} 1$ & 15 & 4.10 & 11.07 & 11 \\
\hline 4. Dieto terapia & $4^{\circ} 1$ & 13 & 3.56 & 9.61 & 10 \\
\hline 5. Nutrición, Actividad Física y Deporte & $6^{\circ} 2$ & 13 & 3.56 & 9.61 & 10 \\
\hline 6. Nutrición en la niñez y adolescencia & $2^{\circ} 1$ & 15 & 4.10 & 11.07 & 11 \\
\hline 7. Seminario de Investigación I & $7^{\circ} 1$ & 16 & 4.38 & 11.82 & 12 \\
\hline 8. Economía Política y Alimentaria & $3^{\circ} 1$ & 13 & 3.56 & 9.61 & 10 \\
\hline $\begin{array}{l}\text { 9. Optativa II: Conservación de alimentos, envase } \\
\text { y embalaje }\end{array}$ & $8^{\circ} 1$ & 15 & 4.10 & 11.07 & 11 \\
\hline 10.Psicología Aplicada a la Nutrición & $4^{\circ} 2$ & 20 & 5.47 & 14.76 & 15 \\
\hline 11. Antropometría & $2^{\circ} 2$ & 9 & 2.46 & 6.64 & 7 \\
\hline 12. Emprendimiento & $8^{\circ} 2$ & 12 & 3.28 & 8.85 & 9 \\
\hline 13.Salud pública y nutrición & $2^{\circ} 3$ & 15 & 4.10 & 11.07 & 11 \\
\hline 14.Técnicas culinarias & $3^{\circ} 1$ & 13 & 3.56 & 9.61 & 10 \\
\hline 15.Soporte Nutricional & $6^{\circ} 3$ & 16 & 4.38 & 11.82 & 12 \\
\hline 16.Normas de Salud y Legislación Sanitaria & $4^{\circ} 3$ & 10 & 2.73 & 7.37 & 7 \\
\hline 17.Administración de Servicios Alimentarios & $6^{\circ} 4$ & 13 & 3.56 & 9.61 & 10 \\
\hline 18.Anatomía y Fisiología Humana & $1^{\circ} 2$ & 10 & 2.73 & 7.37 & 7 \\
\hline 19.Ingles Inicial Avanzado & $2^{\circ} 4$ & 15 & 4.10 & 11.07 & 11 \\
\hline $\begin{array}{l}\text { 20.Seminario de Investigación en } \\
\text { Nutrición II }\end{array}$ & $8^{\circ} 3$ & 16 & 4.38 & 11.82 & 12 \\
\hline $\begin{array}{l}\text { 21.Optativa II: Conservación de alimentos y } \\
\text { envase y embalaje }\end{array}$ & $8^{\circ} 4$ & 15 & 4.10 & 11.07 & 11 \\
\hline 22. Temas Selectos en Nutrición Pediátrica & $4^{\circ} 4$ & 11 & 3.01 & 8.12 & 8 \\
\hline
\end{tabular}


Tabla 1: continuación

\begin{tabular}{|l|c|c|c|c|c|}
\hline \multicolumn{1}{|c|}{ Número de Asignatura } & Período & Población & \%Universo & $\begin{array}{c}\text { \% } \\
\text { Muestra }\end{array}$ & Período \\
\hline 23.Métodos de Análisis Estadísticos de Datos & $6^{\circ} 5$ & 14 & 3.83 & 10.34 & 10 \\
\hline 24.Técnicas e Instrumentos de Investigación & $2^{\circ} 5$ & 15 & 4.10 & 11.07 & 11 \\
\hline 25. Métodos de Análisis Estadístico de Datos & $6^{\circ} 6$ & 11 & 3.01 & 8.12 & 8 \\
\hline 26.Gastronomía & $8^{\circ} 4$ & 17 & 4.65 & 12.55 & 12 \\
\hline 27. Bromatología & $4^{\circ} 5$ & 12 & 3.28 & 8.85 & 9 \\
\hline \multicolumn{2}{|c|}{ Total } & & 365 & & 270 \\
\hline
\end{tabular}

Del total de la población, se obtuvo una muestra de 270 estudiantes con un margen de error del 4,0\% y nivel de confianza del 99\%. Para la selección de los estudiantes se realizó un muestreo aleatorio simple en Tabla 2, se muestra la distribución aleatoria de los sujetos seleccionados entre la población objetivo.

Tabla 2. Selección de estudiantes por muestro simple.

\begin{tabular}{|l|l|}
\hline Asignatura & Sujetos participantes \\
\hline 1. Seminario de Investigación en Nutrición II & $7,6,4,10,2,5,8$ \\
\hline 2.Matemáticas Básicas & $10,8,4,7,11,3,9,2$ \\
\hline $\begin{array}{l}\text { 3. Programas de entrenamiento y ejercicio } \\
\text { físico }\end{array}$ & $5,14,3,9,2,10,6,1,7,4,8$ \\
\hline 4. Dieto terapia & $3,1,10,8,12,6,2,11,9,5$ \\
\hline 5. Nutrición, Actividad Física y Deporte & $3,12,10,1,9,6,11,7,5,2$ \\
\hline 6. Nutrición en la niñez y adolescencia & $12,2,9,11,1,13,10,5,8,6,14$ \\
\hline 7. Seminario de Investigación I & $3,10,4,9,8,5,6,15,16,11,1,2$ \\
\hline 8. Economía Política y Alimentaria & $6,2,7,4,10,5,9,1,3,8$ \\
\hline $\begin{array}{l}\text { 9. Optativa II: Conservación de alimentos, } \\
\text { envase y embalaje }\end{array}$ & $7,8,3,14,11,6,12,13,10,9,15$ \\
\hline 10.Psicología Aplicada a la Nutrición & $6,4,17,16,10,8,5,11,7,15,1,2,12,18,14$ \\
\hline 11. Antropometría & $2,3,7,4,5,1,8$ \\
\hline 12. Emprendimiento & $2,12,7,4,3,6,10,5,11$ \\
\hline 13.Salud pública y nutrición & $14,6,2,10,4,1,11,12,15,7,9$ \\
\hline 14.Técnicas culinarias & $6,5,2,10,9,3,11,12,1,7$ \\
\hline 15, Soporte Nutricional & $4,2,3,8,7,11,10,12,14,1,9,5$ \\
\hline 16.Normas de Salud y Legislación Sanitaria & $6,4,7,3,8,9,5$ \\
\hline 17.Administración de Servicios Alimentarios & $9,6,2,7,4,8,3,5,10,12$ \\
\hline 18.Anatomía y Fisiología Humana & $8,10,9,2,1,5,4$ \\
\hline 19.Ingles Inicial Avanzado & $2,13,4,3,6,8,1,14,12,7,10$ \\
\hline 20.Seminario de Investigación en Nutrición II & $7,2,13,14,10,5,11,16,6,4,1,3$ \\
\hline $\begin{array}{l}\text { 21.Optativa II: Conservación de alimentos y } \\
\text { envase y embalaje }\end{array}$ & $3,7,12,4,5,13,6,9,11,1,10$ \\
\hline 22.Temas Selectos en Nutrición Pediátrica & $4,2,10,3,7,11,6,9$ \\
\hline 23.Métodos de Análisis Estadísticos de Datos & $6,5,8,3,11,4,14,13,7,1$ \\
\hline 24. Técnicas e Instrumentos de Investigación & $12,2,7,10,4,6,14,13,11,5,3$ \\
\hline 25. Métodos de Análisis Estadístico de Datos & $1,3,6,8,5,9,2,10$ \\
\hline 26.Gastronomía & $4,5,11,3,7,2,13,14,15,8,10,16$ \\
\hline 27, Bromatología & $11,10,7,6,1,3,5,8,2$ \\
\hline & \\
\hline & \\
\hline & \\
\hline
\end{tabular}

\section{Instrumento}

Para recoger la información de ambas pruebas se utiliza un cuestionario que cubre la conceptualización formulada según las áreas de interés, validado por (Sánchez-Rivas et al., 2019). En Tabla 3, integrado por 20 ítems categorizados en 5 dimensiones (aprovechamiento; motivación; rendimiento; ubicuidad; satisfacción durante la prueba), donde los estudiantes expresan su opinión según valores tipo Likert con 5 opciones $(5=$ Siempre; $4=$ Casi siempre; 3 = A veces; 2 = Poco; 1 = Nunca). El enfoque didáctico de la intervención se sustenta en la microenseñanza posicionada dentro del paradigma práctico reflexivo, como un proceso de enseñanza aprendizaje y una herramienta de investigación para simplificar el estudio de variables de áreas de interés de aprendizaje y habilidades de enseñanza en un plan de evaluación condesando, permitiendo desmenuzar el complejo acto del evaluador en componentes más simples para practicar, ensayar y reflexionar. La fundamentación micro didáctica de las áreas de interés se plantea a partir de los criterios Stanford Fortune et al. (1967) y los eventos de la instrucción de Gagné (1985) una combinación de enfoque conductista y cognitivista que considera los procesos del aprendizaje, el análisis de los resultados del aprendizaje en la dinámica del aprendizaje, dando lugar a una visión más integradora en la que el aprendizaje se concibe como proceso de asociación y como proceso de reestructuración. 
Tabla 3. Fundamentación micro didáctica, criterios Stanford, eventos de aprendizaje y áreas de interés.

\begin{tabular}{|c|c|c|c|}
\hline Apartados & Criterios Stanford & Eventos de la instrucción & $\begin{array}{l}\text { Digitalizado-G/No } \\
\text { digitalizado }\end{array}$ \\
\hline 1.Generales & $\begin{array}{l}\text {-Organización de la lección. } \\
\text {-Objetivos apropiados. } \\
\text {-Buena selección de contenido y } \\
\text { materiales. }\end{array}$ & $\begin{array}{l}\text { Motivación: Es la fase inicial, que } \\
\text { consiste en crear una expectativa } \\
\text { que mueve al aprendizaje y que } \\
\text { puede tener un origen externo o } \\
\text { interno. }\end{array}$ & $\begin{array}{l}\text { Área de interés: Motivación } \\
\text { del estudiante frente a la } \\
\text { prueba de evaluación. V2: } \\
\text { aceptación emocional de la } \\
\text { evaluación percibida por } \\
\text { los estudiantes }\end{array}$ \\
\hline 2.Presentación & $\begin{array}{l}\text {-Comienzo de la clase. } \\
\text {-Presentación del tema con } \\
\text { claridad. } \\
\text {-Claridad de objetivos. } \\
\text {-Gestión de estrategias } \\
\text { motivadoras. } \\
\text {-Evaluación de conocimientos } \\
\text { previos de los a } \\
\text { alumnos formadores necesidades } \\
\text { e interacción }\end{array}$ & $\begin{array}{l}\text { Comprensión: Se denomina así a } \\
\text { la atención del aprendiz sobre lo } \\
\text { que es importante, y consiste en } \\
\text { el proceso de percepción de } \\
\text { aquellos aspectos que ha } \\
\text { seleccionado y que le interesa } \\
\text { aprender. }\end{array}$ & $\begin{array}{l}\text { Área de interés } \\
\text { Aprovechamiento de la } \\
\text { sesión de evaluación. } \\
\text { Definición.V1: optimización } \\
\text { de tiempos y tareas } \\
\text { durante la sesión de } \\
\text { evaluación en el aula }\end{array}$ \\
\hline 3.Desarrollo & $\begin{array}{l}\text { Comunicación verbal y no verbal } \\
\text { de exposición. } \\
\text {-Participación y atención de los } \\
\text { alumnos. } \\
\text {-Compenetración } \\
\text { entre profesores y alumnos. } \\
\text {-Complementos y comunicación. } \\
\text {-Estrategias didácticas utilizadas. } \\
\text {-Instrucciones claras y criterios de } \\
\text { ejecución bien define. } \\
\text {-Mantiene la secuencia del tema }\end{array}$ & $\begin{array}{l}\text { Adquisición y retención: Éste el } \\
\text { momento crucial del proceso de } \\
\text { aprendizaje. Para Gagné esto } \\
\text { significa incidente esencial } \\
\text { porque marca la transición del } \\
\text { no-aprendizaje al aprendizaje. }\end{array}$ & $\begin{array}{l}\text { Rendimiento del estudiante } \\
\text { en la prueba de evaluación. } \\
\text { V3: proceso de aprendizaje } \\
\text { durante la evaluación } \\
\text { percibida por los } \\
\text { estudiantes }\end{array}$ \\
\hline 4.Cierre & $\begin{array}{l}\text {-Recapitula y sintetiza la } \\
\text { información. } \\
\text {-Comprueba el logro de objetivos. } \\
\text {-Uso de evaluación para mejorar y } \\
\text { como refuerzo, tanto para } \\
\text { alumnos como para alumnos para } \\
\text { el maestro. } \\
\text {-Término adecuado de la lección. } \\
\text {-Variedad de procedimientos para } \\
\text { evaluación. }\end{array}$ & $\begin{array}{l}\text { Recuerdo y transferencia: Son } \\
\text { fases que corresponden al } \\
\text { perfeccionamiento del } \\
\text { aprendizaje. El recuerdo hace } \\
\text { posible que la información se } \\
\text { pueda recuperar mientras que la } \\
\text { transferencia permite que se } \\
\text { pueda generalizar lo aprendido, } \\
\text { que se enseñe lo enseñado. } \\
\text { La fase de retroalimentación } \\
\text { corresponde a la instancia de } \\
\text { desempeño, que se constituye en } \\
\text { un parámetro importante del } \\
\text { aprendizaje. La retroalimentación } \\
\text { consiste en el proceso de } \\
\text { confrontación entre las } \\
\text { expectativas y lo alcanzado en el } \\
\text { aprendizaje. De esta manera el } \\
\text { aprendizaje se verifica y se } \\
\text { afirma, se corrige y avanza. }\end{array}$ & $\begin{array}{l}\text { Satisfacción del estudiante. } \\
\text { V5: percepción personal de } \\
\text { los estudiantes sobre la } \\
\text { efectividad didáctica de la } \\
\text { evaluación. }\end{array}$ \\
\hline $\begin{array}{l}\text { 5. (Liu et al., } \\
\text { 2018), }\end{array}$ & Conexión & Redes & $\begin{array}{l}\text { Ubicuidad: Continuidad del } \\
\text { aprendizaje fuera del aula } \\
\text { (Ubicuidad). V4: } \\
\text { generalización del } \\
\text { aprendizaje involucrado en } \\
\text { la evaluación a contextos } \\
\text { fuera de la escuela. }\end{array}$ \\
\hline
\end{tabular}


Al recoger la información de la intervención y proporciona elementos para conocer si existen condiciones para micronizar la evaluación de acuerdo con las áreas de interés. La primera informa sobre la manifestación disruptiva, el nivel de atención y la realización de las tareas; la segunda identifican los niveles de tranquilidad, el papel activo, el interés y lo agradable de la prueba; la tercera verifica el grado de aplicación de los conocimientos adquiridos para encontrar soluciones a los problemas, y que el resultado sea proporcional al esfuerzo, así como la contribución al desarrollo de competencias; la cuarta busca que la prueba pueda ser aplicada en casa y de forma autónoma, que se muestre a la familia y a los compañeros; la quinta verifica la conclusión satisfactoria de la prueba, pero también, que ésta haya sido coherente con la innovación pedagógica asociada a la intervención del profesor durante la prueba.

\section{Procedimiento}

Las preguntas para ambas pruebas fueron formuladas de acuerdo con los criterios del Centro Nacional de Evaluación para la Educación Superior (Ceneval) que presta servicios de evaluación a cientos de escuelas y universidades, proporcionando información confiable sobre los aprendizajes alcanzados por los estudiantes en los diferentes niveles educativos, mediante el Examen General de Egreso y aproximadamente el 50\% no lo prueba, los participan son alumnos que han cursado el $100 \%$ de los créditos, en su caso, los estudiantes del último semestre de la carrera, siempre y cuando la institución formadora lo solicite, y el examen tiene una duración de 3 y 4 horas. Los criterios de niveles de desempeño por área son: Aun no satisfecho (ANS) si se obtienen de 700 a 999 puntos, para el grado Satisfecho (S)1000-1149 y Sobresaliente (SS) 1150-1300, dos puntuaciones con oportunidad de titulación (Nutrición - Ceneval, 2021).

Como ya se mencionó, las sesiones de intervención se desarrollaron considerando el formato de la prueba no digitalizada en papel y la prueba digitalizada gamificada en kahoot en las que se utilizan los tipos de preguntas del Ceneval orientados a la opción múltiple que contienen básicamente dos elementos: la base es una pregunta, enunciado, declaración o gráfico acompañado de una instrucción que plantea explícitamente un problema, y las opciones de respuesta son enunciados, palabras, cifras o combinaciones de números y letras que se relacionan con la base del ítem del examen, donde sólo una opción es correcta y todas las preguntas tienen cuatro opciones de respuesta como en Kahoot. Para la intervención se eligieron diferentes formas de preguntar: a algunos se les pidió que eligieran el complemento de la información, a otros que eligieran un determinado orden, o que eligieran una lista dada de elementos, y a otros que eligieran la relación de columnas correcta. El test y retest recogen información del proceso para determinar si hay o no una reducción de las áreas de interés del proceso de enseñanza-aprendizaje, por lo que se esperó que los estudiantes manifestaran si observan que en el proceso de evaluación final están presentes las áreas de interés (Tabla 4). La sesión de preparación de la prueba de fin de curso tuvo lugar una semana antes de la intervención y concluyó con la formación de un banco de preguntas del que se extrajeron la primera y la segunda prueba. Cabe destacar que se propusieron tres aplicaciones a los alumnos y éstos eligieron kahoot, argumentando que es más divertida, mientras que los investigadores observaron que juega con dimensiones de cambio, espacio y tiempo propiedades de la micronización y de la gamificación.

La versión gratuita de Kahoot no facilitó la inserción de preguntas en texto debido al límite de palabras que presenta, sin embargo en el formato de imagen que proporciona fue posible incorporar preguntas tipo Ceneval para la fase previa o el antes que corresponde a la preparación de cuarenta a cincuenta preguntas por tema, de las cuales se eligieron quince para la fase durante, el formato se aplicó también para las pruebas no digitalizadas, a continuación se presentan algunos ejemplos, el primero corresponde al tema de Economía política y alimentación: Pregunta conceptual directa: Según Peter Rosset, ¿Qué es la soberanía alimentaria? a) Es el derecho de un gobernante a decidir sobre la protección y regulación de la producción agrícola, b) Es la autosuficiencia de un país a través de los productos excedentes, c) Es el derecho de cada pueblo a definir sus propias políticas agrícolas, d) Es la negación del comercio internacional para lograr el abastecimiento alimentario interno de un país. En las opciones de respuesta se utilizaron los incisos.

La segunda pregunta de procedimiento que implica haber realizado una práctica de la asignatura Conservación, envasado y embalaje de alimentos: En la línea de producción de queso manchego se produjo un problema de contaminación bacteriana y este alimento fue enviado al laboratorio de microbiología para su aislamiento y caracterización mediante las siguientes pruebas, excepto: a) Recuento de bacterias mesófilas aerobias, b) Presencia de Listeria monocytogenes, c) Pruebas bioquímicas, d). Recuento de hongos y levaduras. En las opciones de respuesta se utilizaron los incisos. La tercera pregunta, también difícil de insertar, es de la asignatura de Emprendimiento: ¿Cuál es la finalidad del perfil del proyecto? a) Ayuda a garantizar que los miembros de la comunidad o grupo en cuestión comprendan las posibles implicaciones de su propuesta, b) Esta en función de términos de inversión y costos operativos, c) Evita el desperdicio de esfuerzos, d) Influir en la preparación detallada de proyectos incoherentes. La respuesta es todo lo anterior, por lo que se hicieron cuatro combinaciones para ajustarse a las cuatro opciones de respuesta que ofrece kahoot y según el formato del Ceneval. 
Tabla 4. Especificaciones técnicas de la instrumentación de la intervención.

\begin{tabular}{|c|c|}
\hline \multicolumn{2}{|c|}{ Fases de la intervención en evaluación } \\
\hline \multicolumn{2}{|l|}{ Antes: Una semana intervalo con intervalo de 3 días } \\
\hline \multicolumn{2}{|l|}{ Instrucciones para sesión remota en diferido } \\
\hline \multicolumn{2}{|l|}{$\begin{array}{l}\text { Previamente el administrador prepara un concur } \\
\text { cada materia para que los estudiantes investigu } \\
\text { Ceneval. }\end{array}$} \\
\hline \\
\hline \multicolumn{2}{|l|}{ Descargar la aplicación kahoot en su teléfono móvil } \\
\hline \multicolumn{2}{|l|}{ Se organizan en equipos } \\
\hline \multirow{2}{*}{\multicolumn{2}{|c|}{$\begin{array}{l}\text { Se programan las jugadas } \\
\text { El administrador recupera Ic } \\
\text { para cada equipo y premiac }\end{array}$}} \\
\hline & \\
\hline \multirow{2}{*}{\multicolumn{2}{|c|}{$\begin{array}{l}\text { A partir de esta sesión se elabora un banco de preguntas para cada asignatura } \\
\text { Se signaron } 15 \text { preguntas por cada una de las pruebas para la fase durante. }\end{array}$}} \\
\hline & \\
\hline $\begin{array}{l}\text { Durante: Una semana con intervalo de } 3 \text { días } \\
\text { Secuencia didáctica de la prueba no digitalizado (test) }\end{array}$ & $\begin{array}{l}\text { Durante: Una semana con intervalo de } 3 \text { días } \\
\text { Secuencia didáctica de la prueba digitalizada gamificada } \\
\text { (Retest) }\end{array}$ \\
\hline $\begin{array}{l}\text { Indicaciones de atención durante la aplicación de la prueba } \\
\text { no digitalizada en papel. }\end{array}$ & $\begin{array}{l}\text { Indicaciones de atención durante la aplicación de la } \\
\text { prueba digitalizada y gamificada. }\end{array}$ \\
\hline $\begin{array}{l}\text { a) Abrir la sesión de la prueba } \\
\text { Sus objetos o artículos de clase no deben estar en la } \\
\text { paleta del banco. } \\
\text { El teléfono móvil debe guardarse en la mochila. } \\
\text { Usar lápiz y goma de borrar. } \\
\text { Solo se puede beber agua durante la prueba. } \\
\text { Para ir al sanitario pida permiso al administrador y deje la } \\
\text { prueba. }\end{array}$ & $\begin{array}{l}\text { a). Abrir la sesión de prueba } \\
\text { Sus artículos u objeto de clase no deben estar en la } \\
\text { paleta del banco. } \\
\text { Teléfono móvil en mano. } \\
\text { Comprobar el acceso a internet. } \\
\text { Solo puede beber agua durante la prueba. } \\
\text { Puede ir al sanitario, pero debe decírselo al } \\
\text { administrador de antemano y dejar su teléfono. }\end{array}$ \\
\hline $\begin{array}{l}\text { b) Desarrollar la prueba. } \\
\text { Se distribuyen copia de la prueba en papel por estudiante. } \\
\text { c). Cerrar la sesión de la prueba. } \\
\text { El administrador recoge los exámenes para calificarlos y } \\
\text { entregar calificaciones posteriormente. }\end{array}$ & $\begin{array}{l}\text { b). Desarrollar la prueba. } \\
\text { Los estudiantes ingresan a Kahoot.it. } \\
\text { El administrador proporciona el código de entrada a la } \\
\text { prueba. } \\
\text { El administrador proyecta las preguntas de una en una } \\
\text { en la pizarra. } \\
\text { c). Cerrar la sesión de la prueba } \\
\text { El administrador exporta el reporte en Excel para } \\
\text { entregar de inmediato la calificación obtenida a cada } \\
\text { estudiante. }\end{array}$ \\
\hline $\begin{array}{l}\text { Después: Al finalizar la prueba se evalúa la evaluación con el } \\
\text { test de áreas de interés } \\
\text { Se analiza la información } \\
\text { Se integra el informe de resultados }\end{array}$ & $\begin{array}{l}\text { Después: Al finalizar la prueba se evalúa la evaluación con } \\
\text { el retest de áreas de interés } \\
\text { Se analiza la información } \\
\text { Se integra el informe de resultados }\end{array}$ \\
\hline
\end{tabular}

La dificultad de insertar preguntas largas en Kahoot no fue una limitación insuperable, ya que tanto las preguntas como las respuestas se presentaron en imágenes y utilizando afirmaciones cortas en los cuadros de respuesta para la fase de preparación como en la de aplicación de la prueba. Cabe señalar que el cuestionario de evaluación para recoger información de la prueba digitalizada gamificada y de la prueba no digitalizada, se realizó en papel.

\section{RESULTADOS Y DISCUSIÓN}

En la fase del antes, las pruebas duraron entre veinticinco y cincuenta minutos. Para conocer el alcance de la intervención en las calificaciones correspondientes a la etapa del durante se procedió a buscar las tendencias y determinar si las calificaciones están asociadas y en qué dirección (Figura 2). En la Figura 2, el modelado de la prueba no digitalizada en 27 asignaturas reportó duración de quince minutos, donde $\mathrm{y}=4 \mathrm{x}+59$ por cada prueba de asignatura aumentó a 4 la cantidad de estudiantes que obtuvieron 10 sobre la base de 59, el coeficiente de correlación $\mathrm{R}^{2}=0.75$ se aproxima a 1, por lo que es aceptable el modelado. El coeficiente de correlación lineal donde $\mathrm{R}^{2}=0$ es nulo en calificación de 9 . Para el modelado negativo donde $y=4 . x+133$ por cada prueba de materia disminuyen la cantidad de 4 estudiantes que obtienen 8 sobre la base de 133, el coeficiente correlación $\mathrm{R}^{2}=0.74$ se aproxima a 1 , por lo que se acepta el modelado.

En la Figura 3, el modelado de la prueba digitalizada gamificada de la intervención en 27 asignaturas reportó duración de cinco minutos, donde $\mathrm{y}=6 \mathrm{x}+204$ por cada prueba de asignatura disminuyó la cantidad de 6 estudiantes que obtienen 8 sobre la base de 204, el coeficiente correlación $\mathrm{R}^{2}=0.93$ se aproxima a 1 por lo 
que es aceptable el modelado negativo. En ecuación donde $y=3 x+11$ por cada prueba de asignatura aumento a 3 la cantidad de estudiantes que obtuvieron 9 sobre la base de 11 , el coeficiente de correlación $\mathrm{R}^{2}=0.89$ se aproxima a 1, por lo que es aceptable el modelado. La ecuación en donde y $=3 x+56$ por cada prueba de asignatura aumentó a 3 la cantidad de alumnos que obtuvieron 10 con base en 55, el coeficiente de correlación $\mathrm{R}^{2}=0.81$ se aproxima a 1 , por lo que es aceptable el modelado. El test y retest proporcionan información para el primer análisis descriptivo de la fase después que se realiza para obtener una aproximación a la naturaleza de la información, ya que se ha identificado la desviación estándar con respecto a la media Figura 4, permite visualizar cómo fluctúan las medias en su conjunto.

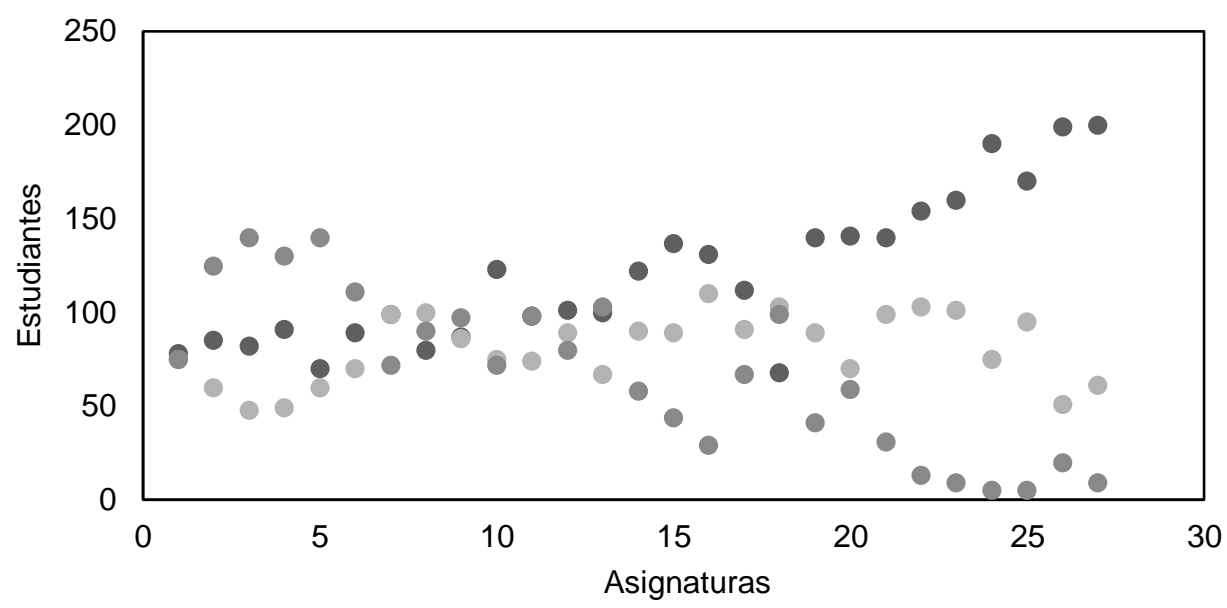

Fig. 2. Cantidad de estudiantes y calificaciones en prueba no digitalizada. Los símbolos corresponden a calificaciones: • 9.75-10, • 8.7-9.02, • 8-8.35.

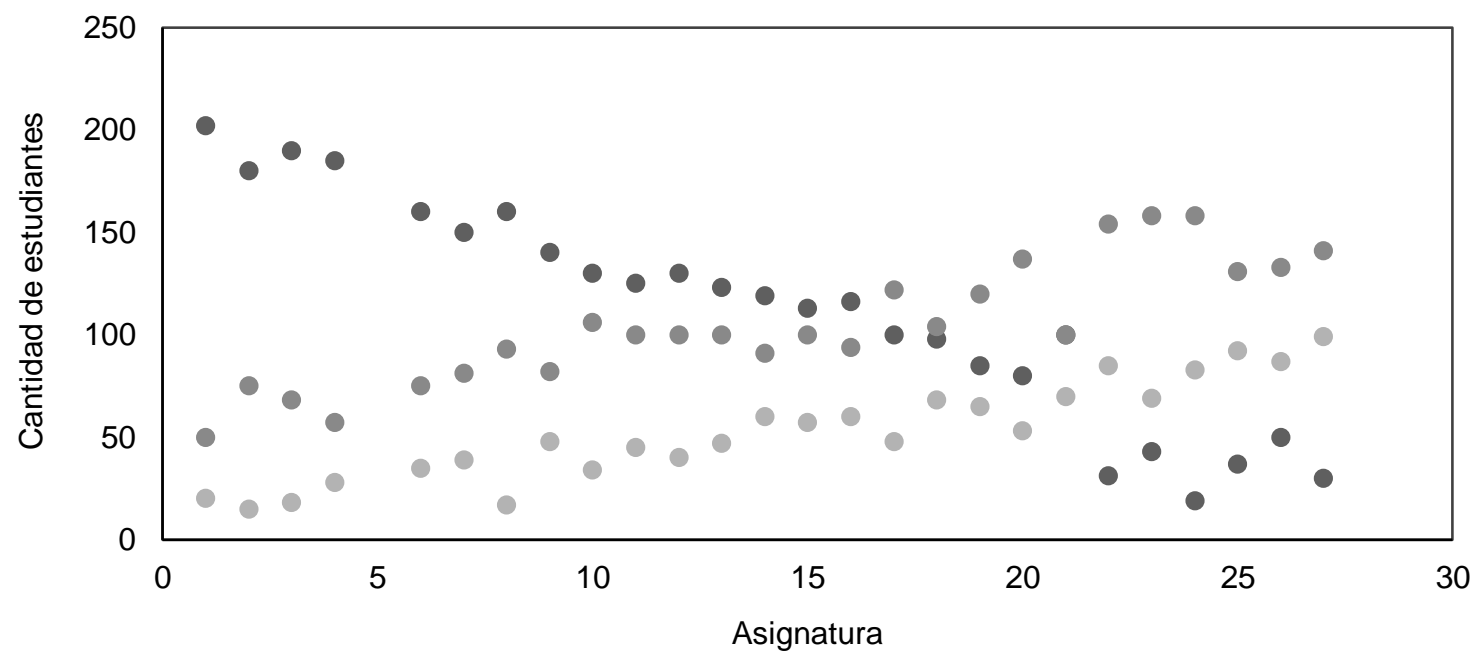

Fig. 3. Cantidad de estudiantes y calificaciones de prueba digitalizada gamificada. Los símbolos corresponden a calificaciones: • 8.-35, • 8.7-9, • 9.75-10.

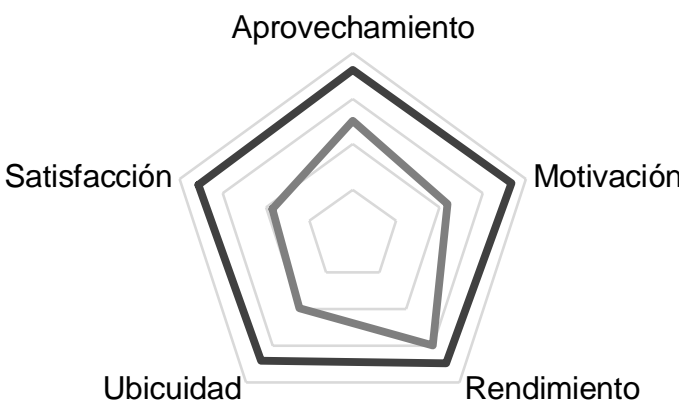

Fig. 4. Polígonos de medias de 5 áreas en 27 materias diferentes en ambas pruebas. Símbolos: test a la prueba no digitalizada -, retest a la prueba digitalizada gamificada - 
El Figura, 4 en el primer bloque los ítems del 1 al 12 los estudiantes valoraron el aprovechamiento de la sesión de evaluación, la motivación y el rendimiento durante la prueba de evaluación; el segundo bloque ítems del 13 al 16 valoraron la continuidad del aprendizaje fuera del aula indicada como ubicuidad; el tercer bloque de ítems 17 al 20 valoraron el área de interés estratégica estrechamente vinculada al alumno y al profesor en la búsqueda de una mejor experiencia personal como la satisfacción. El test y retest computa datos del durante de la sesión de evaluación en puntuaciones medias en un rango de 0-20 y respectiva desviación estándar que dan cuenta de la presencia de las cinco áreas de interés, según la prueba digitalizada y gamificada: aprovechamiento 18.15-1.61, motivación- 18.33-1.42, rendimiento 17.4.-2.50 ubicuidad 19-2, satisfacción 17.83-1.19. Según la prueba no digitalizada: aprovechamiento 12.56-2-04, motivación 10.94-1.97, rendimiento14.98-3.58, ubicuidad 9.93-1.04 y satisfacción 9.29-1.80, con mayor media y menos desviación estándar para la prueba digitalizada gamificada.

La simplificación ocurre cuando las medias de la prueba no digitalizada y la prueba digitalizada gamificada forman polígonos, aunque son irregulares mantienen una orientación similar sin cruce de líneas, y la altura de los puntos se mantiene dentro de la forma produciendo la inclusión del ángulo, confirmando simplificación en ambas pruebas, mientras que la condición de las variables se visualiza en Figura 5.

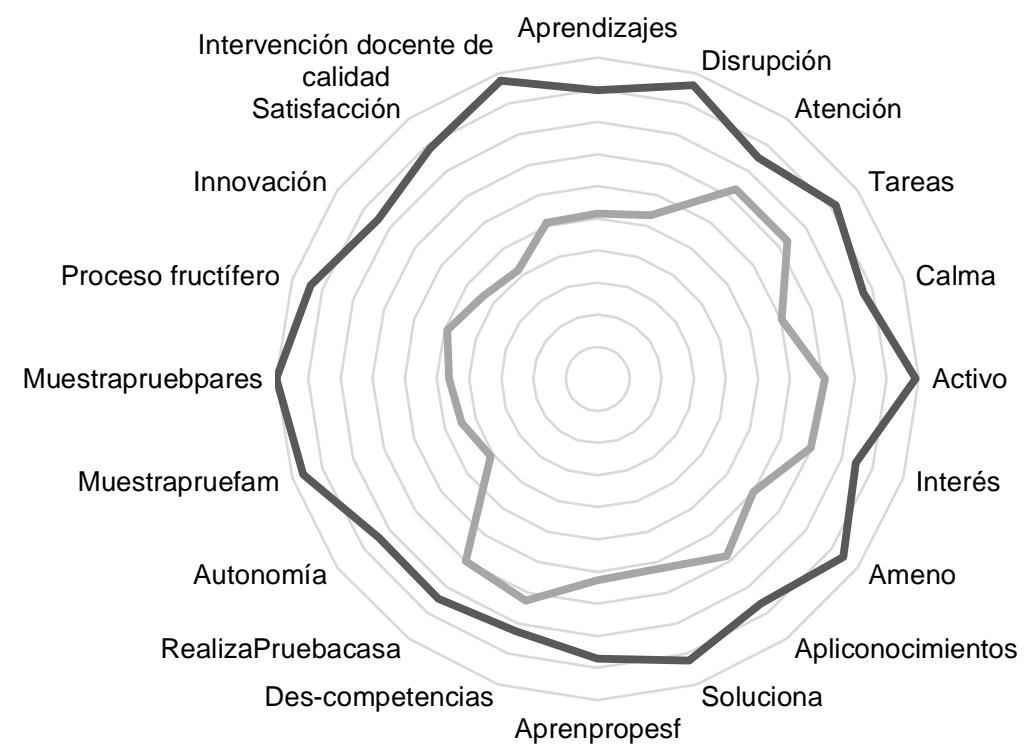

Fig. 5. Polígono de variables de las áreas de interés, símbolos: test de la prueba digitalizada gamificada -, retest de la prueba no digitalizada -

La simplificación también ocurre en las variables de las áreas de interés según Figura 5 , en la que se visualiza la condición interna simplificada, en un polígono más regular para la digitalizada gamificada y menos regular para el no digitalizada, sin cruce de líneas. En este sentido la información corresponde a un durante con un intervalo de una semana, por lo que al explorarse por primera vez su temporalidad es desconocida, y puede interpretarse como el estado en que han operado y operan. Esta visualización se basa en las medias y desviaciones obtenidas para las variables de las áreas de interés, de las cuales la primera es el aprovechamiento: 1. Que durante la aplicación de la prueba se realicen aprendizajes, en la prueba no digitalizada (nd) de acuerdo al test, (nd) Media-2.57, DS-0.83; prueba digitalizada gamificada (dg) de acuerdo al retest, Media-4.49, SD-0.64; 2. Que la manifestación disruptiva haya sido escasa nd 2.68-0.54, dg 4.810.38; 3. Que se haya mantenido un buen nivel de atención nd 3.65-1.00, dg 4.26-0.66; 4. Que el estudiante haya realizado todas las tareas previstas nd 3.65-0.62, dg 4.59-0.51.

Para motivación: 5. Que el estudiante manifieste calma hacia la prueba de evaluación nd 3.01-0.41, dg 4.35$0.84 ; 6$. Que el estudiante adopte un papel activo en la tarea nd 3.54-1.14, dg 4.95- 0.22; 7. Que el estudiante manifieste interés hacia la prueba de evaluación nd 3.49-0.65, dg 4.23-0.74; 8. Que el estudiante manifieste que el proceso de evaluación fue ameno nd 3-56, dg 4.72-0.45. Para rendimiento:9. Que el estudiante aplique los conocimientos adquiridos con éxito nd 3.41-1.19, dg 4.32- 0.62; 10. Que el estudiante encuentre soluciones a los problemas planteados nd 3.1-0.74, dg 4.61-0.48; 11 . Que el resultado del aprendizaje sea proporcional al esfuerzo nd 3.13-0.84, dg 4.35-0.65; 12. Que contribuya al desarrollo de competencias clave nd 3.63-1.30, dg 4.13-1.05. 
Para ubicuidad: 13. Que el estudiante haya aplicado la prueba de evaluación en su casa nd 3.50-0.43, dg 4.23-0.64; 14. Que el estudiante haya manejado la prueba de evaluación de forma autónoma nd 2.05-0.58, dg 4.21-0.39; 15. Que el estudiante haya mostrado la prueba de evaluación a su familia nd 2.22-0.66, dg 4.820.38; 16. Que el estudiante haya mostrado la prueba de evaluación a su grupo de pares nd 2.31-0.61, dg 500. Para satisfacción: 17. Que el proceso de evaluación haya sido fructífero para el estudiante nd 2.46-0.71, dg 4.70-0.60; 18. Que la evaluación sea coherente con la innovación pedagógica nd 2.20-0.71, dg 4.22-0.76; 19. Que la sesión de evaluación haya terminado de forma satisfactoria nd 2.09-0.80, dg 4.43-0.72; 20. Que la intervención docente haya sido de calidad nd 2.55-1.12, dg 4.88-0.64, con mayor media y menos desviación estándar para la prueba digitalizada gamificada.

A la luz de este informe se utiliza el estadístico Kolmogórov-Smirnov con la corrección de Lilliefors para obtener resultados más precisos sobre la significación, la varianza, y comprobar si el comportamiento en las medias evidencia normalidad de los datos. Dado que existe una muestra superior a 30 o 50, la prueba es viable. En la Tabla 5, la función de distribución de los datos muestra que el p-valor es inferior al nivel de significancia 0,05 , por lo que se rechaza la hipótesis alternativa $\mathrm{H}_{1}$ al presentar dos formatos de prueba, y uno de ellos poco usual, se encontraría diferencias significativas, y se acepta la hipótesis nula $\mathrm{H}_{0}$ al presentar dos formatos de prueba, y uno de ellos poco usual, no se encontraría diferencias significativas, asumiendo que se cumplen niveles de medición y no se cumplen los requisitos paramétricos y considerando que la falta de significancia en los datos no determina que las mediciones no tengan una base para establecer relaciones, se procede a utilizar la prueba de correlación no paramétrica Rho de Spearman la cual revela una correlación positiva alta en áreas de interés de aprovechamiento, motivación y rendimiento, y baja en ubicuidad y satisfacción prevaleciendo significancia inexistente, por lo que se realiza un análisis de tendencias para determinar de manera las áreas de interés se asocian y en qué dirección, Figura 6.

Tabla 5. Prueba de normalidad de los datos.

\begin{tabular}{|l|l|l|l|}
\hline \multirow{2}{*}{ Área } & \multirow{2}{*}{ Tipo } & \multicolumn{2}{|c|}{ Kolmogórov-Smirnov } \\
\cline { 3 - 4 } & & \multicolumn{1}{c|}{ Coeficiente } & \multicolumn{1}{c|}{ Sig. } \\
\hline \multirow{2}{*}{ Aprovechamiento de la sesión de evaluación. } & No digitalizado & .19 & .00 \\
\cline { 2 - 4 } & Digitalizado & .26 & .00 \\
\hline Motivación de los frente a la prueba de evaluación. & No digitalizado & .22 & .00 \\
\cline { 2 - 4 } & Digitalizado & .22 & .00 \\
\hline \multirow{2}{*}{ Rendimiento de los estudiantes en la prueba. } & No digitalizado & .17 & .00 \\
\cline { 2 - 4 } & Digitalizado & .21 & .00 \\
\hline Continuidad del aprendizaje fuera del aula (Ubicuidad). & No digitalizado & .20 & .00 \\
\cline { 2 - 4 } & Digitalizado & .35 & .00 \\
\hline Satisfacción del estudiante. & No digitalizado & .14 & .00 \\
\cline { 2 - 4 } & Digitalizado & .20 & .00 \\
\hline
\end{tabular}

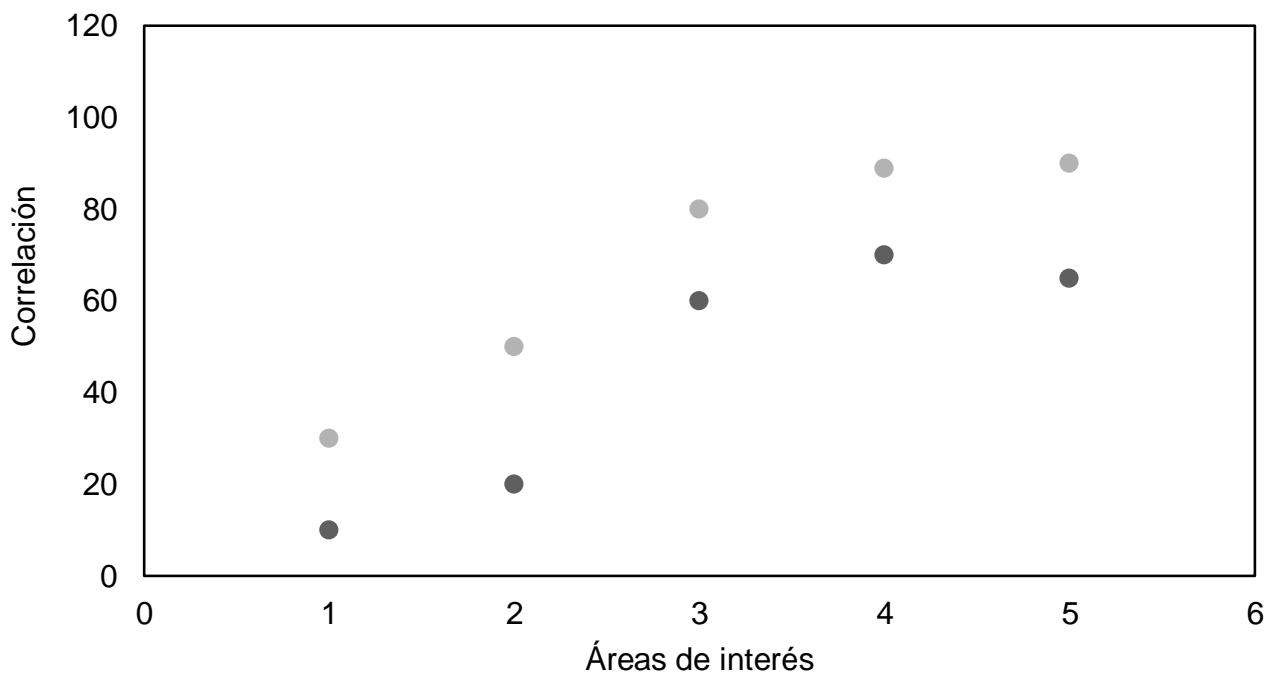

Fig. 6. Correlación y áreas de interés. Los símbolos corresponden a calificaciones: test la prueba no digitalizada $\bullet$, retest de la prueba digitalizada y gamificada $\bullet$. 
En Figura 6, el modelado del test y retest presenta dos variantes correspondientes a la fase durante, una en la prueba digitalizada gamificada donde $y=16 x+20$ por cada área de interés aumento la correlación en 16 puntos con base en 20, el coeficiente de correlación $\mathrm{R}^{2}=0.89$ se aproxima a 1 , por lo que es aceptable el modelado. En la prueba no digitalizada donde $y=16 x-3$ indica que por cada área de interés la correlación aumento 16 puntos menos 3 , el coeficiente de correlación $\mathrm{R}^{2}=0.82$ se aproxima a 1 , por lo que se acepta el modelado. Cabe señalar que el modelado micronizado aporta elementos que validan la transposición didáctica de las áreas de interés, los criterios Stanford (Allen, 1967) y los eventos de la instrucción de (Gagné, 1985) en la formulación del concepto micro evaluación.

El modelado de calificaciones en 27 asignaturas obtenido de la fase durante de la cual el resultado del test y retest contribuyó a la innovación de la prueba de fin de curso, también revela que existen posibilidades de influir en la preparación y la predicción de resultados respecto al examen EGEL. Asimismo, este es un resultado que contribuye a la factibilidad y viabilidad de micronizar el formato de reactivos EGEL ya que los contenidos que abarca son amplios, por ejemplo, para la formación en Nutrición cubre tres áreas con once subáreas. El modelado de las calificaciones del durante y el modelado de la correlación de las áreas de interés del después aportaron elementos para afirmar que ocurrió micronización durante el proceso de evaluación. Por lo tanto, se puede concluir que se ha obtenido un modelo micronizado de evaluación de fin de curso, reiterando que fue posible innovar la prueba de fin de curso, que siempre se ha aplicado de la misma manera en la UAT desde que legisló sobre ella.

La fundamentación micro didáctica de las áreas de interés del proceso de enseñanza-aprendizaje en los criterios de Stanford y los eventos instruccionales de Gagné permitieron incorporar a las áreas de interés el componente de ubicuidad y situarla dentro del proceso de enseñanza aprendizaje en la perspectiva de la actual red interconectada de Internet. Sin embargo, hay que reconocer que la población estudiada, a pesar de vivir interconectada, no lo percibieron con relevancia, como también ocurrió con la satisfacción, mientras que, al aprovechamiento, la motivación y el rendimiento se les dio importancia según el modelado de correlación.

Fue sustancial que las herramientas mediante las cuales se realizó la función simplificadora mantuvieran el carácter y la forma de las áreas de interés del proceso de enseñanza aprendizaje en la evaluación no digitalizada durante quince minutos y en la evaluación digitalizada gamificada durante cinco minutos, tiempo con el que la evaluación se integra a la familia de los micros: la microenseñanza (Allen (1967), la microdocencia Dulsat-Ortiz (2019), el modelo microcurricular activo de Posso-Pacheco et al. (2020) y se vincula a la gamificación por medio del microlearning que se sirve de la socialización mediática y las identidades lúdicas que lo convierten en multisensorial Hug (2007) y multimodal Dolasinski y Reynolds (2020).

Las microevaluación obtenida se puede clasificar en más regular y menos regular, es probable que la prueba micronizada no digitalizada corresponda en general a la clasificación de menos regular, mientras que la prueba micronizada digitalizada gamificada corresponde a la clasificación de más regular. La acción pedagógica permitió movilizar al alumno en el centro de la evaluación, vinculando el enfoque micro orientado a la didáctica, la no digitalización, la digitalización y la gamificación para generar la simplificación y lograr la microactualización de la evaluación. Que hayan dado los estudiantes cuenta en el test y retest de la percepción de que en el proceso de evaluación también se desarrollan áreas de interés del proceso de enseñanza aprendizaje valida la micronización de los contenidos, y que hayan conseguido un tiempo de cinco y quince minutos validando la micronización del tiempo como ocurre en la metodología para microenseñanza de (Allen, 1967 y Fortune et al., 1967). El modelado revela que tanto la gamificación como la microenseñanza son dos metodologías que contribuyeron a micronizar la evaluación. Una portó las dimensiones de cambio, tiempo espacio, y la otra aportó la estructura didáctica y el contenido micro orientado, constituyendo un binomio para simplificar áreas de interés en la evaluación, ya que estas pertenecen de forma general al proceso de enseñanza aprendizaje.

La intervención en veintisiete asignaturas de la Licenciatura en Nutrición suma varios estudios que dan cuenta de la introducción de la gamificación en el ámbito disciplinar de la educación superior (Tsay et al., 2018 y Zabala-Vargas et al., 2020). Así como su papel en la formación que involucra los dispositivos móviles y otras aplicaciones (Jaimes, 2020 y Morales et al., 2020). La Kahoot permitió jugar un proceso con las variables tiempo, espacio de contenido y cambio que no sólo evitaron la disminución de la atención, sino que favorecieron la concisión, la fragmentación del contenido en pequeñas unidades, la reducción del tiempo registrado en dos minutos dulces y agradables, seis minutos en los que decae el compromiso y la sentencia lo nivela, y doce minutos en los que las exigencias pueden ser dulces y agradables.

El modelado se sitúa en la corriente principal de evaluación gamificada como evaluación gamificada digitalizada, y no digitalizada (Zabala-Vargas et al., 2020). En contexto del contenido micro orientado se identifica como evaluación micronizada, ambas variantes del modelado de correlación se concretan en un proceso de evaluación de la evaluación como lo reconocen Sepúlveda et al. (2020). 
La evaluación micronizada se enmarca en una época donde las conexiones, la velocidad de la información y la comunicación empujan a la priorización y ésta a su vez a la micronización "pequeña y rápida" casi a nivel de un teaser que dura trece segundos jugando con las emociones y la mente de los usuarios resaltando características, o el tráiler con duración de dos minutos suficientes para proporcionar una sinopsis audiovisual de un video musical, serie, película, cortometraje o videojuego. Con las reservas necesarias, la similitud en educación puede ser el microlearning, el más buscado para apoyar procesos de enseñanza y aprendizaje en diversos ámbitos formativos. Son contextos donde el juego privilegia uno o varios canales sensoriales y se presenta como una solución, sin embargo, en este caso no es así, ya que el foco no estuvo centrado en el juego acabado y concreto, sino en la gamificación apoyada en fundamentos pedagógicos y didácticos para el cambio, el tiempo y el espacio que contribuyeron a perfilar el proceso de reflexión sobre la evaluación plagada de oportunidades, fortalezas, obstáculos o debilidades que hay que gestionar (González-Barea et al., 2021).

A manera de síntesis la intervención consideró al menos dos sesiones previas de socialización gamificadas de conocimientos para ambas pruebas y al aplicarse la prueba micronizada no digitalizada los estudiantes requirieron de 15 minutos, mientras que para la prueba digitalizada y gamificada de 5 minutos. Se promovió que cada participante construyera su propia perspectiva sobre las variables de las áreas de interés basados en sus propias experiencias y esquemas mentales desarrollados. El instrumento de evaluación de la evaluación se aplicó en un promedio de 3 a 5 minutos de duración. Finalmente, coincidimos con Sepúlveda et al. (2020) en la importancia de la evaluación como proceso, y con Allen (1967) al percibir la microenseñanza como una metodología que aborda la mejora de procesos. Con Tsay et al. (2018) y Jaimes (2020) entre otros en que la gamificación también contribuye a la conducción de procesos evaluativos.

\section{CONCLUSIONES}

La evaluación micronizada pretende difundir la importancia de evaluar la evaluación en la Universidad Autónoma de Tamaulipas. Al replicar la intervención en la prueba de fin de curso, supondría una innovación ya que la forma de presentarlas reglamentada es únicamente oral y escrita. Esta metodología también es útil para contribuir a la concientización de la importancia de la evaluación como un proceso y no como una actividad más que debe realizarse en el momento, fecha y lugar indicados, que además equivale al $50 \%$ de la calificación global del estudiante. Asimismo, se alcanzó a micronizar la prueba de preparación para la realización del examen EGEL. Cabe señalar que no se ha intentado reproducir los ítems de la prueba EGEL, sólo el formato de las preguntas. Como conclusión principal, podemos hablar de preproceso, proceso y postproceso de la evaluación una vez validadas las fases en la modelización que dieron lugar a la evaluación micronizada.

\section{REFERENCIAS}

Alhalafawy, W. S., y Zaki, M. Z., The Effect of Mobile Digital Content Applications Based on Gamification in the Development of Psychological Well-Being, https://doi.org/10.3991/ijim.v13i08.10725, International Journal of Interactive Mobile Technologies (iJIM), 13(08), 107-123 (2019)

Allen, D. W., Micro-Teaching: A New Framework for In-Service Education, The High School Journal, ISSN 00181498, 49(8), 355-362 (1966)

Castañeda-Vázquez, C., Espejo-Garcés, T., Zurita-Ortega, F., y Fernández-Revelles, A., La formación de los futuros docentes a través de la gamificación, tic y evaluación continua, https://doi.org/10.6018/sportk.391751, SPORT TKRevista EuroAmericana de Ciencias del Deporte, 8(2), 55-63 (2019)

Centro Nacional para la Evaluación de la Educación Superior (CENEVAL)., Guía para el sustentante Examen General para el Egreso de la Licenciatura en Nutrición. https://ceneval.edu.mx/wp-content/uploads/2021/07/EX-EGELGUIA_EGEL_NUTRICION_20210715.pdf (2021)

Chevallard, Y., Readjusting Didactics to a Changing Epistemology, https://doi.org/10.2304/eerj.2007.6.2.131, European Educational Research Journal, 6(2), 131-134 (2007)

Dolasinski, M. J., y Reynolds, J., Microlearning: A new model of learning, https://doi.org/10.1177/1096348020901579, Journal of Hospitality \& Tourism Research, 44(3), 551-561 (2020)

Dulsat-Ortiz, C., Microenseñanza en el laboratorio de ciencias para el alumnado del grado de educación infantil, https://doi.org/10.14483/23448350.14769, Revista Científica, 3(36), 367-380 (2019)

Fortune, J. C., Cooper, J. M., y Allen, D. W., The Stanford Summer Micro-Teaching Clinic, 1965, https://doi.org/10.1177/002248716701800402, Journal of Teacher Education, 18(4), 389-393 (1967)

Fuentes-Cabrera, A., Parra-González, M. E., López-Belmonte, J., y Segura-Robles, A., Learning Mathematics with Emerging Methodologies-The Escape Room as a Case Study, https://doi.org/10.3390/math8091586, Mathematics, 8(9), 1586 (2020)

Gagné, R. M., The Conditions of Learning and Theory of Instruction, 4ª Ed., 331-349, Wadsworth Pub Co, ISNB 9780030636882 California, USA (1985) 
Galazzi, L., Gómez, D., y Vázquez, M., Políticas mundializadas de formación docente: Propuestas para una lectura filosófica, https://doi.org/10.19053/22160159.v10.n22.2019.9308, Praxis \& Saber, 10(22), 19-43 (2019)

González-Acosta, E., Almeida-González, M., Torres-Chils, A., y Traba-Montejo, Y. M., La gamificación como herramienta educativa: el estudiante de contabilidad en el rol del gerente, del contador y del auditor, https://doi.org/10.4067/S0718-50062020000500155, Formación Universitaria, 13(5), 155-164 (2020)

González-Barea, E. M., Rodríguez-Entrena, M. J., y Minyety-Baez, M. E., Evaluación de la educación universitaria en República Dominicana, https://doi.org/10.4067/S0718-50062021000200013, Formación Universitaria, 14(2), 13-24 (2021)

Gutiérrez-Monsalve, J. A., Garzón, J., y Segura-Cardona, A. M., Factores asociados al rendimiento académico en estudiantes universitarios, https://doi.org/10.4067/S0718-50062021000100013, Formación Universitaria, 14(1), 13-24 (2021)

Huang, R., Ritzhaupt, A. D., y otros seis autores, The impact of gamification in educational settings on student learning outcomes: A meta-analysis, https://doi.org/10.1007/s11423-020-09807-z, Educational Technology Research and Development, 68(4), 1875-1901(2020)

Hug, T., Didactics of Microlearning, 1ra. Ed., 35-53 Waxmann Publishing Co, ISBN 978-3-8309-1869-1 New York, USA (2007)

Ibañez, M. B., Di-Serio, A., y Delgado-Kloos, C., Gamification for Engaging Computer Science Students in Learning Activities: A Case Study, https://doi.org/10.1109/TLT.2014.2329293, IEEE Transactions on Learning Technologies, 7(3), 291-301 (2014)

Isabelle, D. A., Gamification of Entrepreneurship Education, https://doi.org/10.1111/dsji.12203, Decision Sciences Journal of Innovative Education, 18(2), 203-223 (2020)

Jaimes, A., La Feria Lúdico-Pedagógica como espacio de reflexión curricular, https://doi.org/10.4067/S071850062020000400057, Formación Universitaria, 13(4), 57-68 (2020)

Kelleci, O., Kulaksiz, T., y Pala, F. K., The effect of social network-supported microteaching on teachers self-efficacy and teaching skills, https://doi.org/10.18844/wjet.v10i2.3456, World Journal on Educational Technology: Current Issues, 10(2), 115-130 (2018)

Kohnke, L., y Ting, A., ESL students' perceptions of mobile applications for discipline-specific vocabulary acquisition for academic purposes, https://doi.org/10.34105/j.kmel.2021.13.006, Knowledge Management \& E-Learning: An International Journal, 13(1), 102-117 (2021)

Liu, M., Huang, Y., y Zhang, D., Gamification's impact on manufacturing: Enhancing job motivation, satisfaction and operational performance with smartphone-based gamified job design, doi.org/10.1002/hfm.20723, Human Factors and Ergonomics in Manufacturing \& Service Industries, 28(1), 38-51 (2018)

Morales, J. C., Ramírez, N. E., Vargas, S. H., y Peñuela, A. J., Uso de aplicativos móviles en el aula y sus factores determinantes, https://doi.org/10.4067/S0718-50062020000600013, Formación Universitaria, 13(6), 13-22 (2020)

Pérez, L. I., Rivera, G, E., y Delgado, F. M., Mejora de hábitos de vida saludables en alumnos universitarios mediante una propuesta de gamificación, https://doi.org/10.20960/nh.669, Nutrición Hospitalaria, 34(4), 942-951 (2017)

Posso-Pacheco, R. J., Barba-Miranda, L. C., y otros cuatro autores, Modelo de aprendizaje microcurricular activo: Una guía de planificación áulica para Educación Física, https://doi.org/10.15359/ree.24-3.14, Revista Electrónica Educare, 24(3), 1-18. (2020)

Sailer, M., y Sailer, M., Gamification of in-class activities in flipped classroom lectures, https://doi.org/10.1111/bjet.12948, British Journal of Educational Technology, 52(1), 75-90 (2021)

Sánchez-Rivas, E., Ruiz-Palmero, J., y Sánchez-Rodriguez, J., Gamification of Assessments in the Natural Sciences Subject in Primary Education, https://doi.org/10.12738/estp.2019.1.0296, Educational Sciences: Theory \& Practice, 19, (1), 95-111 (2019)

Sepúlveda, A., Minte, A., Canales, R., y Díaz-Levicoy, D., Dimensiones cognitivas en los procesos de evaluación en carreras pedagógicas, https://doi.org/10.4067/S0718-50062020000500129, Formación Universitaria, 13(5), 129-136 (2020)

Tsay, C. H., Kofinas, A., y Luo, J., Enhancing student learning experience with technology-mediated gamification: An empirical study, https://doi.org/10.1016/j.compedu.2018.01.009, Computers \& Education, 121, 1-17 (2018)

Zabala-Vargas, S. A., Ardila-Segovia, D. A., García-Mora, L. H., y Benito-Crosetti, B. L., Aprendizaje Basado en Juegos (GBL) aplicado a la enseñanza de la matemática en educación superior. Una revisión sistemática de literatura, https://doi.org/10.4067/S0718-50062020000100013, Formación Universitaria, 13(1), 13-26 (2020) 